\title{
E OS HOMENS? E OS QUE MORAM LONGE? E OS MAIS JOVENS? ...? PERFIL DOS USUÁRIOS DE PROGRAMAS DE ATIVIDADE FÍSICA OFERECIDOS PELAS UNIDADES BÁSICAS DE SAÚDE DE LONDRINA-PR
}

\author{
DR. MATHIAS ROBERTO LOCH \\ Departamento de Educação Física, Centro de Educação \\ Física e Esporte, Universidade Estadual de Londrina \\ (Londrina - Paraná - Brasil) \\ E-mail: mathias@uel.br
}

\begin{abstract}
GRAD. CAMILA GUERREIRO RODRIGUES
Bacharelado em Educação Física, Departamento de Educação Física, Centro de Educação Física e Esporte, Universidade Estadual de Londrina (Londrina - Paraná - Brasil) E-mail: camilaguerreirol3@yahoo.com.br
\end{abstract}

\author{
DR. DENILSON DE CASTRO TEIXEIRA \\ Departamento de Educação Física, Centro de Educação \\ Física e Esporte, Universidade Estadual de Londrina \\ (Londrina - Paraná - Brasil) \\ E-mail: denict@uel.br
}

\begin{abstract}
RESUMO
A criação do Núcleo de Apoio à Saúde da Família (NASF) representou um importante avanço para o sistema de saúde brasileiro, bem como uma importante oportunidade de inserção profissional da Educação Física. O objetivo do estudo foi verificar o perfil dos usuários de programas de atividade física oferecidos pelo NASF de Londrina (PR). Realizou-se entrevista com todos os participantes de programas de atividade física oferecidos em 5 Unidades Básicas de Saúde ( $n=167)$. Em geral, a participação ficou restrita às mulheres, pessoas com mais de 50 anos, que já apresentam algum tipo de doença e que moram próximas aos locais onde eram realizados os programas. Faz-se necessária a criação de estratégias mais abrangentes para aumentar a chance de a população se tornar fisicamente ativa.
\end{abstract}

PALAVRAS-CHAVE: Sistema de Saúde; promoção da saúde; atividade física; saúde da família. 
A partir, principalmente, da segunda metade do século $X X$, vários países, inclusive o Brasil, passaram por um importante processo de transição demográfica e epidemiológica. Estas transformações resultaram no envelhecimento populacional e no aumento das doenças crônicas não transmissíveis causadas pela diminuição da taxa de natalidade, aumento da expectativa de vida, e a diminuição da participação relativa das doenças infectocontagiosas como causa de morbidade e mortalidade (BUCHALLA; WALDMAN; LAURENT, 2003; SCHRAMM et al., 2004; SCHIMIDT, et al., 20 I I).

Paralelo a esta modificação epidemiológica, estudos confirmaram a antiga suposição de que indivíduos fisicamente ativos apresentavam menor incidência de determinadas doenças, de mortalidade por todas as causas e por algumas causas específicas, que seus pares menos ativos (LEE; BLAIR, 2002; SCHNOHR; SCHARLING; JENSEN, 2003). Entretanto, apesar dos indicadores que apontam a prática de atividade física como um importante comportamento relacionado à saúde, a adoção deste comportamento envolve questões mais amplas, não sendo, na maioria dos casos, o conhecimento da importância da prática suficiente para que indivíduos e populações sejam de fato fisicamente ativos.

Nesse sentido, o Ministério da Saúde do Brasil tem buscado ressaltar meios e estratégias que auxiliem a prática de atividade física regular por parte da população brasileira. Dentre tais estratégias, no início de 2008 foram criados os Núcleos de Apoio à Saúde da Família (NASF), que devem buscar sua atuação seguindo algumas diretrizes relacionadas à atenção primária, entre os quais a ação interdisciplinar e intersetorial, a educação permanente em saúde (tanto da população, quanto dos profissionais de saúde), a integralidade, o desenvolvimento de território, a participação social, a educação popular, a promoção da saúde e a humanização. Sendo assim, potencialmente o NASF pode ser importante no sentido de ampliar a diversidade das ações das Equipes de Saúde da Família, bem como aumentar a resolubilidade dos problemas de saúde da população (BRASIL, 2009).

Um dos profissionais que pode fazer parte das equipes do NASF é o de Educação Física. Sua intervenção deve ser embasada na reflexão e organização de diferentes estratégias que objetivem elevar o nível de atividade física da população. Devido à recente implantação do NASF, pouco ainda se sabe sobre o perfil dos usuários que utilizam estes serviços. Assim, devido à escassez de informações a respeito deste contexto, os estudos que envolvam a Educação Física neste campo de atuação são necessários, inclusive para se avaliar o nível de cobertura das ações. Com base na problemática levantada, o objetivo deste estudo foi verificar o perfil dos usuários de programas de atividade física oferecidos pelas Unidades Básicas de Saúde atendidas por profissionais de Educação Física, pertencentes ao NASF do município de Londrina, Paraná. 


\section{MÉTODOS}

\section{SELEÇÃO DA AMOSTRA}

No NASF de Londrina, município localizado na Região Norte do Paraná e que tem mais de 500 mil habitantes, estavam inseridos, no segundo semestre de 2010, 10 profissionais de Educação Física. Para a composição da amostra, foram sorteadas de maneira aleatória, através do programa Randomizer (disponível em www.randomizer.com), cinco das 33 Unidades Básicas de Saúde (UBS) da região urbana de Londrina/PR. Após o sorteio foi realizado contato prévio e agendada reunião com os profissionais das UBS sorteadas, para esclarecimento sobre os objetivos do estudo e os procedimentos que seriam utilizados.

Foram selecionados todos os sujeitos que realizavam atividade física regularmente, há pelo menos um mês, nos grupos das UBS sorteadas, sendo este o único critério de inclusão. Grupos que apenas recebiam orientações sobre atividade física (como por exemplo: grupos de gestantes, diabéticos, hipertensos, etc.) não foram incluídos na amostra.

\section{PROCEDIMENTOS PARA COLETA E ANÁLISE DOS DADOS}

Anteriormente à coleta de dados, foi realizado o esclarecimento aos sujeitos sobre os objetivos e procedimentos que seriam adotados no estudo, deixando claro que a participação na pesquisa era voluntária. Os participantes que optaram por participar do estudo assinaram Termo de Consentimento Livre e Esclarecido.

Para a coleta das informações, levando-se em conta os objetivos do estudo, foi criado um questionário, que foi avaliado e aprovado por três professores com experiência na área de atividade física e saúde. Este instrumento continha questões relacionadas a informações pessoais, característica de participação no programa, tempo e forma de deslocamento até o programa, participação em práticas de atividade física fora do programa, reconhecimento do profissional responsável pelo programa, e sobre a presença de doenças, massa e estatura corporal e autopercepção de saúde.

O questionário foi aplicado em forma de entrevista individual por uma única pesquisadora, nos dias e locais em que eram realizadas as atividades físicas oferecidas pelas UBS. A coleta foi realizada nos meses de agosto e setembro de 2010.

A coleta de dados teve seu início após a aprovação do Comitê de Ética em Pesquisa com Seres Humanos da Universidade Estadual de Londrina (Parecer 062/I 0).

Os dados foram tabulados no Programa Excel para Windows e analisados no Programa SPSS versão 19.0. Para a análise dos dados utilizou-se elementos da estatística descritiva. 


\section{RESULTADOS}

Participaram deste estudo 167 sujeitos, com média de idade de 62,6 $(\mathrm{DP}=\mathrm{I} \mathrm{I}, \mathrm{I})$ anos. Não houve recusas em participar do estudo. Na Tabela I, em que são apresentadas as características demográficas da amostra, bem como a prevalência de sobrepeso e obesidade e a percepção de saúde dos sujeitos, observou-se que a maior parte dos sujeitos tinha mais que 50 anos $(90,4 \%)$, eram mulheres $(89,8 \%)$, casados (59,3\%), não haviam completado o ensino fundamental $(75,4 \%)$, apresentavam sobrepeso ou obesidade (64,6\%) e percebiam sua saúde como boa (62,3\%).

$\mathrm{Na}$ Tabela 2, em que são apresentados os resultados referentes à participação no programa, observa-se que quase metade dos sujeitos $(48,5 \%)$ referiu ter tomado conhecimento através de amigos. $\bigcirc$ tempo de participação dos usuários no programa se mostrou bastante diverso, variando de um mês (que era o critério de inclusão) até 24 meses. Em relação à frequência semanal ao programa, a maior parte referiu participar em até dois dias semanais (61,1\%). Quanto ao grau de satisfação dos sujeitos com o programa, todos avaliaram o programa como muito bom ou bom. Nenhum dos entrevistados referiu insatisfação com o programa.

Tabela I. Características demográficas, Índice de Massa Corporal e Percepção de Saúde

\begin{tabular}{ccc}
\hline Variável & $n$ & $\%$ \\
\hline Sexo & & \\
Feminino & 150 & 89,8 \\
Masculino & 17 & 10,2 \\
Faixa Etária & & \\
Até 39 anos & 5 & 3,0 \\
40 a 49 anos & 11 & 6,6 \\
50 a 59 anos & 43 & 25,7 \\
60 a 69 anos & 67 & 40,2 \\
70 anos ou mais & 41 & 24,5 \\
Estado civil & & \\
Solteiro & & 3,6 \\
Casado & 6 & 59,3 \\
Viúvo & 99 & 28,7 \\
Divorciado & 48 & 8,4 \\
\hline Grau de Escolaridade & 14 & \\
Nunca estudou & & 16,2 \\
\hline Variável & & continua
\end{tabular}


Ensino Fundamental incompleto

99

59,2

Ensino Fundamental Completo/Médio incompleto

15

9,0

Ensino Médio completo/Superior incompleto

$17 \quad 10,1$

Ensino Superior completo

9

5,4

Índice de Massa Corporal

Normal $\quad 58$

35,2

Sobrepeso

70

42,2

Obesidade

37

22,4

Percepção de saúde

Ótima

26

15,6

Boa

104

62,3

Regular

33

19,8

Ruim

4

2,4

Tabela 2. Características da participação no programa (como ficou sabendo, tempo de participação, frequência de participação e grau de satisfação)

\begin{tabular}{ccc}
\hline Variáveis & $\mathrm{n}$ & $\%$ \\
\hline Como ficou sabendo & 8 Amigos & 48,5 \\
Anúncio na igreja & 15 & 8,9 \\
Agente de saúde & 13 & 7,8 \\
Médico & 12 & 7,2 \\
Unidade Básica de Saúde & 12 & 7,2 \\
Viu as pessoas fazendo os exercícios & 10 & 5,9 \\
Família & 7 & 4,2 \\
Profissional de Educação Física & 5 & 2,9 \\
Grupo de Idosos do Bairro & 4 & 2,3 \\
Cartaz de divulgação & 3 & 1,8 \\
Outros & 5 & 2,9 \\
Há quanto tempo participa? & & \\
I a 3 meses & & 27,0 \\
4 a 6 meses & 45 & 19,8 \\
7 a 9 meses & 33 & 6,6 \\
I0 a I2 meses & 11 & 19,8 \\
Mais de I2 meses & 33 & 27,0 \\
& 45 & \\
Quantas vezes por semana participa? & & 36,5 \\
I vez & 61 & 24,6 \\
\hline
\end{tabular}

continua 


\begin{tabular}{ccc}
\hline Variáveis & $\mathrm{n}$ & $\%$ \\
\hline 3 vezes & 63 & 37,7 \\
4 vezes & 2 & 1,6 \\
& & \\
Grau de satisfação & & 54,5 \\
Muito Bom & 91 & 45,5 \\
Bom & 76 & \\
\hline
\end{tabular}

Tabela 3. Tempo e forma de deslocamento utilizado até o local do programa

\begin{tabular}{ccc}
\hline Minutos & $\mathrm{n}$ & $\%$ \\
\hline $0-5$ & 86 & 51,5 \\
$6-10$ & 54 & 32,3 \\
$11-15$ & 18 & 10,8 \\
$16-20$ & 8 & 4,8 \\
$21-25$ & 0 & 0 \\
$26-30$ & 1 & 0,6 \\
\hline
\end{tabular}

Forma de deslocamento

\begin{tabular}{ccc}
\hline Meio utilizado & $\mathrm{n}$ & $\%$ \\
\hline Caminhando & 156 & 93,4 \\
Ônibus & 7 & 4,2 \\
Carro & 3 & 1,8 \\
Bicicleta & $\mathrm{I}$ & 0,6 \\
\hline
\end{tabular}

Na Tabela 3 constam as informações referentes ao tempo de deslocamento dos sujeitos até o local do programa. Foi observado que a média de tempo de deslocamento foi de 7,82 minutos ( $D P=5, I$ minutos). Pouco mais da metade das pessoas levava menos que cinco minutos para chegar até o local dos exercícios $(51,5 \%)$, sendo que quase todos os sujeitos $(93,4 \%)$ referiram chegar até o local das atividades caminhando.

Em relação às informações sobre prática de atividades físicas fora do programa da UBS, a Tabela 4 mostra que metade dos sujeitos investigados (50,3\%) relatou participar de outras atividades físicas, sendo que destes, 51,2\% faziam caminhada por conta própria. Outras pessoas mencionaram participar de programas oferecidos por universidades, farmácias e supermercados ou em grupos de idosos. Havia ainda aquelas que realizavam aulas em academias, aulas de dança de salão, natação, pilates, exercício físico em casa ou participam das atividades oferecidas por outra UBS. Em relação ao conhecimento dos sujeitos sobre qual profissional tem a responsabilidade 
pela prescrição dos exercícios físicos, a maioria (73,7\%) apontou o profissional de Educação Física como sendo o responsável.

Tabela 4. Participação dos usuários do programa oferecido pelas UBSs em outras atividades físicas e conhecimento sobre a formação do profissional responsável

\begin{tabular}{|c|c|c|}
\hline Variáveis & $\mathrm{n}$ & $\%$ \\
\hline \multicolumn{3}{|c|}{ Prática atividade física fora do Programa } \\
\hline $\operatorname{Sim}$ & 84 & 50,3 \\
\hline Não & 83 & 49,7 \\
\hline \multicolumn{3}{|l|}{ Tipo de atividade física } \\
\hline Caminhada & 43 & 51,2 \\
\hline Grupo de idosos do Bairro & 12 & 14,2 \\
\hline Universidades & 8 & 9,5 \\
\hline Farmácias & 6 & 7,1 \\
\hline Academia & 5 & 6,0 \\
\hline Outra UBS & 4 & 4,7 \\
\hline Supermercados & 4 & 4,7 \\
\hline Em casa & 2 & 2,3 \\
\hline Pilates & । & 1,2 \\
\hline Natação & । & 1,2 \\
\hline \multicolumn{3}{|l|}{ Dança de Salão } \\
\hline & I & 1,2 \\
\hline \multicolumn{3}{|c|}{$\begin{array}{l}\text { Profissional responsável pelo Programa } \\
\text { Profissional de Educação Física }\end{array}$} \\
\hline Fisioterapeuta & 127 & 73,7 \\
\hline Enfermeiro & 15 & 9,0 \\
\hline Médico & 11 & 6,6 \\
\hline \multirow[t]{2}{*}{ Não sabe } & 3 & 1,8 \\
\hline & 15 & 9,0 \\
\hline
\end{tabular}

Sobre a prevalência de pessoas que possuíam doenças, bem como os tipos de doenças autorrelatadas (TABELA 5), 78,4\% dos sujeitos possuíam algum tipo de doença. As mais prevalentes foram hipertensão (52,7\%) e diabetes $(20,4 \%)$.

Tabela 5. Prevalência de doenças autorrelatadas nos indivíduos participantes do programa

\begin{tabular}{ccc}
\hline Doenças & $\mathrm{n}$ & $\%$ \\
\hline Sim & 131 & 78,4 \\
Não & 36 & 21,5 \\
& & \\
Doença & & \\
\hline
\end{tabular}




\begin{tabular}{ccc}
\hline Doenças & $\mathrm{n}$ & $\%$ \\
\hline Hipertensão & 88 & 52,7 \\
Diabetes & 34 & 20,4 \\
Colesterol & 28 & 16,8 \\
Tireoide & 12 & 7,18 \\
Artrose & 10 & 6,0 \\
Problemas de coluna & 6 & 3,6 \\
Depressão & 5 & 3,0 \\
Arritmia & 4 & 2,4 \\
Sinusite & 4 & 2,4 \\
Artrite & 4 & 2,4 \\
Bico de papagaio & 4 & 2,4 \\
Reumatismo & 4 & 2,4 \\
Osteoporose & 3 & 1,8 \\
Asma & 3 & 1,8 \\
AVC & 3 & 1,8 \\
Desgaste na coluna & 2 & 1,2 \\
Labirintite & 2 & 1,2 \\
Outros & 14 & 8,4 \\
\hline
\end{tabular}

Outros inclui: bursite (I), cardiopatia (I), catarata (I), insônia (I), chagas (I), tendinite no ombro (I), deficiência na perna $(\mathrm{I})$, derrame no olho $(\mathrm{I})$, DPOC $(\mathrm{I})$, faringite $(\mathrm{I})$, gastrite $(\mathrm{I})$, hérnia de disco $(\mathrm{I})$, infarto $(\mathrm{I})$, problemas renais $(\mathrm{I})$.

\section{DISCUSSÃO}

Este trabalho teve como objetivo verificar o perfil dos usuários de programas de atividade física oferecidos pelas Unidades Básicas de Saúde atendidas por profissionais de Educação Física pertencentes ao NASF de Londrina, que é o município com maior população da região Norte do Paraná. Conhecer este perfil se faz necessário no sentido de que a oferta de atividade física nas UBS é recente, e que possivelmente a população em geral ainda associa mais o trabalho nas UBS na lógica médico/hospitalar/curativa do que como um espaço também para a prevenção de doenças e da promoção da saúde.

Através dos resultados obtidos no presente estudo observou-se que a maioria dos usuários do programa de atividade física oferecido pelas UBS eram mulheres. Este resultado é praticamente o mesmo observado por Hallal et al. (20 I 0) que, na ocasião, constataram que 89,2\% dos usuários do Programa Academia da Cidade da cidade do Recife (Pernambuco), eram do gênero feminino (contra 89,8\% do presente estudo).

Parte desta diferença em relação à participação dos gêneros pode estar relacionada aos horários e dias das atividades, tendo em vista que a proporção 
de homens que trabalham fora de casa é superior à de mulheres (OLIVEIRA; SCORZAFAVE; PAZELLO, 2009), o que pode representar um fator que dificulta a adesão ao programa por parte dos homens, uma vez que os programas são oferecidos em horário comercial.

De qualquer forma, esta maior procura/participação por parte das mulheres não se dá exclusivamente em programas de atividade física oferecidos pelos serviços públicos. Parece evidente que, em geral, as mulheres procuram com maior frequência os serviços preventivos de saúde. A baixa procura de homens a esses serviços levou o Ministério da Saúde a criar a Política Nacional de Atenção Integral à Saúde do Homem, que objetiva uma maior conscientização por parte da população masculina em relação à importância do autocuidado com a saúde (BRASIL, 2008).

Em relação à forma como os participantes tomaram conhecimento do programa, quase metade referiu ter sido por intermédio de amigos. Chama atenção a baixa proporção de sujeitos que soube do programa por profissionais das respectivas UBS. Isso pode demonstrar baixo comprometimento e/ou conhecimento dos profissionais envolvidos na equipe da UBS na divulgação das atividades físicas oferecidas pelo programa. Estes achados concordam com os de Siqueira et al. (2009b), que demonstraram ser baixa a prevalência de aconselhamento educativo para a prática de atividade física entre usuários das UBSs, nas regiões Sul e Nordeste do Brasil. Na ocasião, os autores concluíram ser necessária melhorar a participação dos profissionais de diferentes áreas, para que a condução de aconselhamentos relacionados à prática de atividade física seja mais efetiva. Não se pode também descartar, que a dinâmica de trabalho (inclusive pelo excesso de trabalho) seja também um fator que comprometa uma melhor divulgação do programa.

O alto nível de satisfação dos sujeitos com o programa de atividades físicas parece deixar clara a boa avaliação por parte dos usuários. Entretanto, é preciso observar este dado com cautela, uma vez que alguns indivíduos podem ter avaliado o programa de maneira positiva com receio de que os resultados desta pesquisa prejudicassem de alguma forma a continuidade do programa, haja vista a instabilidade dos profissionais e dos programas e alguns problemas recentes ocorridos com o NASF no município de Londrina (problemas estes que resultaram inclusive na paralisação total das atividades em algumas semanas em 2009). Outra hipótese para o alto nível de satisfação encontrado pode estar relacionado ao perfil da amostra: maioria mulheres, parte considerável com mais de 60 anos e baixo nível de escolaridade. Para Neri (2007), as mulheres com esse perfil tendem a afirmar-se pela atividade, participação, independência e autonomia para gerir as suas vidas e para frequentar locais onde antes a sua presença era incomum. A "libertação" da mulher com a idade avançada de certos controles sociais como a reprodução e cuidado 
com os filhos, permitem que elas ocupem esses espaços (grupos de convivência, de atividade física, etc.) como símbolo de liberdade. Assim, considerando o ineditismo das experiências vividas nessas atividades, fazem com que muitas dessas mulheres vivenciem a atividade com grande motivação. A falta de experiências em situações similares pode fazer com que essas mulheres tenham dificuldades em avaliar esse tipo de programa mais criticamente.

De qualquer modo, mesmo com estas ponderações, não deixa de ser um dado positivo que nenhum dos entrevistados tenha avaliado o programa de maneira negativa (regular, ruim ou muito ruim). Esses achados são coerentes com os divulgados pelo Instituto de Pesquisa Econômica e Aplicada (IPEA), que demonstrou que a maior parte dos usuários está satisfeita com os serviços públicos e saúde no Brasil. Na citada pesquisa, o Programa Saúde da Família foi avaliado como muito bom ou bom por $80,7 \%$ da população (BRASIL, 20I I ). Fato curioso é que na pesquisa do IPEA os sujeitos que haviam utilizado o SUS nos meses anteriores à pesquisa avaliaram de maneira mais positiva os serviços do que aqueles não usuários recentes do SUS.

Foi observado no estudo que pouco mais da metade dos indivíduos levam até cinco minutos $(51,5 \%)$ para chegar ao local do programa, que geralmente tem suas atividades desenvolvidas nas proximidades das UBS. Isso pode ser um indicativo de que as atividades desenvolvidas pelos profissionais de Educação Física acabam tendo uma área de abrangência pequena, ficando restrita à população que reside nas proximidades do local onde é realizada a prática de atividades físicas.

Travassos e Martins (2004) revisaram várias dimensões relacionadas ao acesso e utilização dos serviços de saúde. Entre elas estão as dimensões sócio-organizacionais, que se refere às características da oferta de serviços, e a geográfica, que reflete a distância percorrida pelos usuários até a obtenção dos serviços de saúde. Neste sentido, parece claro que a participação nos programas será mais provável nas pessoas que moram próximas aos locais de prática e para as quais os horários ofertados são convenientes. Evidentemente, não são apenas estes aspectos que determinarão a adesão ou não por parte da população aos programas. Sabe-se que a prática de atividade física é um comportamento complexo e que muitos outros fatores devem ser considerados.

Foi elevada a proporção de sujeitos que reconheceu o profissional de Educação Física como o responsável pelos programas de atividade física. Este é um dado importante, uma vez que muitas vezes a Educação Física ainda é associada somente à prática esportiva ou como disciplina escolar, sendo relativamente recente a aproximação desta área, pelo menos de uma maneira mais sistemática, com a saúde pública, inclusive porque a inserção do profissional de Educação Física nos programas de pós-graduação em Saúde Coletiva e Epidemiologia, passou a ocorrer com maior frequência somente a partir dos anos 2000 (HALLAL; KNUTH, 20 I I). 
A alta prevalência de doenças e de excesso de peso nos indivíduos demonstra que estes, talvez, busquem os serviços de saúde, bem como a atividade física ofertada pela UBS, quando já possuem alguma doença instalada, fenômeno este que tem sido referido como causalidade reversa. Por exemplo, estudo de Dumith, Domingues e Gigante (2009) observou que entre indivíduos adultos da cidade de Pelotas, Rio Grande do Sul, os obesos apresentaram maior probabilidade de caminharem no tempo livre do que aqueles com peso considerado normal, muito provavelmente por que tinham a prática desta atividade como um dos fatores importantes para a redução do peso corporal.

De qualquer modo, nosso achado é relevante no sentido que a lógica do NASF é - ou deveria ser - principalmente a da prevenção da doença e da promoção da saúde. Assim, as ações devem atender toda a comunidade e não estarem restritas aos indivíduos mais vulneráveis ou já adoecidos.

Mesmo com a alta proporção de sujeitos que referiram possuir algum tipo de doença, foi observado que 77,9\% dos sujeitos percebiam a sua saúde como ótima ou boa. Esse dado corrobora com Siqueira et al. (2009a) que, investigando adultos e idosos das regiões Sul e Nordeste, observaram que a maior parte dos sujeitos apresentava percepção positiva de saúde (na ocasião os autores ainda observaram que a maior proporção de percepção de saúde positiva foi encontrada entre os sujeitos fisicamente ativos). Além disso, os achados indicam que muitas pessoas superaram a noção dicotômica da saúde entendida meramente como ausência de doença (do contrário, as pessoas com doença perceberiam de maneira negativa sua própria saúde). Fonseca et al. (20 I0) investigaram idosos e observaram que, para eles, a autonomia tinha um papel mais relevante na autoavaliação da saúde, do que a presença ou não de doenças. Vale mencionar que a prática regular de atividade física pode ser importante no sentido de retardar os agravos relacionados ao processo de envelhecimento e aumentar a autonomia funcional das pessoas.

Diante dos desafios e dificuldades que a implantação do NASF tem enfrentado, é clara a importância que este programa possui para a saúde pública do Brasil. Talvez o maior problema a ser superado seja a quebra do paradigma do atendimento individual, ampliando assim o enfoque para uma lógica coletiva, o que concorda com o ponto de vista de Florindo (2009). Um dos pontos fortes é a equipe multiprofissional que integra o NASF, o que deve permitir o desenvolvimento de ações pautadas nos princípios da integralidade e universalidade do atendimento à saúde da família.

Outra questão a ser destacada é que os municípios estão em diferentes estágios de implementação do NASF, sendo que em alguns, o NASF se encontra em um estágio de consolidação mais avançado, enquanto que em outros sequer existe. De qualquer modo, a atuação do profissional de Educação Física no NASF 
não deve se restringir à criação de grupos de atividade física. Estudo de Souza e Loch (20 I I) investigou a característica da intervenção do profissional de Educação Física no NASF da região norte do Paraná, e observou que a maior parte tem uma ação limitada aos grupos. Neste sentido, faz-se necessário que a intervenção profissional desenvolva o empoderamento das comunidades e promova a participação de pessoas e organizações, no sentido de que os sujeitos e comunidades tenham real controle sobre suas vidas.

Uma possível dificuldade para que as ações não sejam tão restritas, se deve pela própria formação profissional. Apesar de não ser problema exclusivo da Educação Física, alguns trabalhos demonstram e discutem estas dificuldades (PASQUIM, 20 I 0; ANJOS; DUARTE, 2009) nesta área específica do conhecimento, apontando que a formação ainda é hegemonicamente centrada na lógica individual e biológica, havendo pouco espaço para conteúdos relacionados à saúde coletiva.

Mesmo considerando algumas limitações e delimitações do presente estudo, entre os quais estão o delineamento transversal da pesquisa e o número de sujeitos, que ficou limitado aos participantes de cinco das 33 UBS de Londrina/PR, vale destacar que ainda são poucos os estudos desta natureza, e que os achados deste podem fornecer elementos importantes para a reflexão sobre as possibilidades e limitações da atuação do profissional de Educação Física no contexto do NASF, principalmente quando este se limita à organização de grupos de prática de atividade física.

\section{CONCLUSÃO}

De maneira sucinta, este trabalho demonstrou que a participação nos programas de atividade física oferecidos pelo NASF de Londrina, PR, está em grande medida restrito a pessoas com certas características, a saber: mulheres, com mais de 50 anos, com baixa escolaridade, que possuem pelo menos uma doença, que tem excesso de peso e que moram perto do local de realização das atividades.

Considerando o atual estágio do conhecimento na área da atividade física relacionada às ações de saúde pública, e a recente implementação do NASF, as informações aqui apresentadas podem contribuir tanto para as intervenções no programa quanto para o direcionamento de ações que aumentem a abrangência do mesmo para a população em geral, uma vez que os dados reforçam a ideia de que as ações baseadas exclusivamente em programas formais de atividades físicas acabam atingindo apenas pessoas com um perfil bem específico. Apesar da importância em atender este público, pessoas com outras características também podem usufruir benefícios do trabalho da equipe do NASF e da Estratégia Saúde da Família. 
Além do mais, para melhorar a cobertura das ações dentro dos próprios programas, faz-se necessária a criação de outros locais de prática (uma possibilidade é alternar os locais onde acontece a atividade), para que as populações que moram mais afastadas possam também se beneficiar de tal serviço, ou mesmo que seja ofertado transporte aos sujeitos que residem mais afastados. Sugere-se ainda que os horários em que são ofertadas as atividades sejam repensados, principalmente no sentido de oportunizar esta prática aos indivíduos que não possuem disponibilidade em horário comercial.

\section{Where Are the Men? Where Are the People who Live far Away? Where Are the Young?....? Profile of Users of Physical Activity Programs Offered by Family Health Suport Centers}

The creation of the Family Health Suport Centers (FHSC) represented an important advance for the Brazilian health system, and an important opportunity for Physical Education. The objective of this study was to determine the profile of users of physical activity programs offered by the FHSC in a city in southern Brazil. We conducted interviews with all participants in physical activity programs offered in five Primary health care units $(n=167)$. In general, participation in physical activity was restricted to women, people over 50 years, people who already have some kind of disease and who live close to where the programs are conducted. The strategies related in context to FHSC to physical activity not to be limited to the creation of practice groups. It is necessary that broader strategies are developed.

KEYWORDS: Primary Health Care; Health Promotion; Physical Activity; Family Health Program.

\section{¿Dónde están los hombres? ¿Dónde están aquellos que viven lejos?} ¿Dónde están los jóvenes? ...? Perfil de los usuarios de programas de actividad física de las Unidades Básicas de Salud de Londrina-PR

La creación del Centro de Apoyo a la Salud de la Familia (NASF) representó un importante avanzo para el sistema de salud brasileño, así como una importante oportunidad para la integración del profesional de Educación Física. El objetivo del estudio fue determinar el perfil de los usuarios de programas de actividad física que ofrecen las Unidades Básicas de Salud (UBS) de Londrina (PR). Realizamos entrevistas con todos los participantes en programas de actividad fisica que se ofrecen en cinco UBS $(n=167)$. Se observó que la participación en programas de actividad física es en gran parte restringido a las mujeres, las personas mayores de 50 años, que ya tienen algún tipo de enfermedad y que viven cerca de los sitios donde los programas se llevan a cabo. Es necesario crear estrategias más amplias para aumentar las oportunidades para que las personas sean activas físicamente.

PALABRAS CLAVE: Sistema de Salud; promoción de la salud; actividad fisica; salud de la familia. 


\section{REFERÊNCIAS}

ANJOS, T. C.; DUARTE, A. C. G. A Educação Física e a estratégia saúde da família: formação e atuação profissional. Physis Revista de Saúde Coletiva, Rio de Janeiro, v. 19 , n. 4, p. I 127 | | 44, 2009.

BRASIL. Ministério da Saúde. Secretaria de Atenção a Saúde. Política Nacional de Atenção Integral à Saúde do Homem: princípios e diretrizes. Brasília, 2008.

BRASIL. Ministério da Saúde. Secretaria de Atenção à Saúde. Departamento de Atenção Básica. Cadernos de Atenção Básica, n. 27. Brasília, 2009.

BRASIL. Instituto de Pesquisa Econômica Aplicada. SIPS: Sistema de Indicadores de Percepção Social. 09 fev. 201 I. Disponível em: <http://unw.ipea.gov.br/portal/images/stories/PDFs/ SIPS/I 10207 sipssaude.pdf>. Acesso em: 7 jul. 201 I.

BUCHALLA, C. M.; WALDMAN, E. A.; LAURENTI, R. A mortalidade por doenças infecciosas no início e no final do século $X X$ no município de São Paulo. Revista Brasileira de Epidemiologia, São Paulo, v. 6, n. 4, p. 335-344, dez. 2003.

DUMITH, S. C.; DOMINGUES, M. R.; GIGANTE, D. P. Epidemiologia das atividades físicas praticadas no tempo de lazer por adultos do Sul do Brasil. Revista Brasileira de Epidemiologia, São Paulo, v. 12, n. 4, p. 646-656, dez. 2009.

FLORINDO. A. A. Núcleos de apoio à saúde da família e a promoção das atividades físicas no Brasil de onde viemos onde estamos e para onde vamos. Revista Brasileira de Atividade Física e Saúde, Pelotas, v. 14. n. 2, p. 72-73, 2009.

FONSECA, M. G. U. P et al. Papel da autonomia na auto-avaliação da saúde do idoso. Revista de Saúde Pública, São Paulo, v. 44 n. I p. 159-65, fev. 2010.

HALLAL, P. C. et al. Avaliação do Programa de Atividade Física Academia da Cidade de Recife, Pernambuco, Brasil: percepções de usuários e não usuários. Cadernos de Saúde Pública, Rio de Janeiro, v. 26, n. I p. 70-78, jan. 2010.

HALLAL, P. C.; KNUTH, A. G. A epidemiologia da atividade física e a aproximação necessária com as pesquisas qualitativas. Revista Brasileira de Ciências do Esporte. Florianópolis, v. 33, n. I, p. 181-192, mar. 201।.

LEE C. D.; BLAIR S. N. Cardiorespiratory fitness and stroke mortality in men. Medicine Science in Sport and Exercise, Hagerstown, v. 34, n. 4 p. 592-595, apr. 2002.

NERI, A. L. Qualidade de vida e envelhecimento na mulher. In: (Org.). Desenvolvimento e envelhecimento: perspectivas biológicas, psicológicas e sociológicas. Campinas: Papirus, 2007. OLIVEIRA, P. R.; SCORZAFAVE, L. G.; PAZELLO, E. T. Desemprego e inatividade nas metrópoles brasileiras: as diferenças entre homens e mulheres. Nova Economia, Belo Horizonte, v. 19, n. 2, p. 291-324, maio/ago. 2009. 
PASQUIM, H. M. A saúde coletiva nos cursos de graduação em Educação Física. Saúde e Sociedade, São Paulo, v. 19, n. I p. 193-200, jan./mar. 2010.

SCHIMIDT, M. I. et al. Chronic non-communicable diseases in Brazil: burden and current challenges. Lancet (British edition), Londres, v. 377, p. 1949-1961, 201 I.

SCHRAMM, J. M. A. et al. Transição epidemiológica e o estudo de carga de doença no Brasil. Revista Ciência e Saúde Coletiva, Rio de Janeiro, v. 9, n. 4, p. 897-908, 2004.

SCHNOHR, P.; SCHARLING, H.; JENSEN, J. S. Changes in Leisuretime Physical Activity and risk of death: na observational study of 7000 men and women. American Journal Epidemiology, v. 158, n. 7, p. 639-644, oct. 2003.

SIQUEIRA, F. V. et al. Fatores considerados pela população como mais importantes para manutenção da saúde. Revista de Saúde Pública, São Paulo, v. 43, n. 6, p. 961 -7I, jan. 2009a.

SIQUEIRA, F. V. et al. Aconselhamento para a prática de atividade física como estratégia de educação à saúde. Cadernos de Saúde Pública, Rio de Janeiro, v. 25, n. I , p. 203-21 3, jan. 2009 b.

SOUZA, S. C.; LOCH, M. R. Intervenção do profissional de educação física nos núcleos de apoio à saúde da família em municípios do norte do Paraná. Revista Brasileira de Atividade Física e Saúde, Pelotas, v. 16, n. I, p. 5-10, 2011.

TRAVASSOS, C.; MARTINS, M. Uma revisão sobre os conceitos de acesso e utilização de serviços de saúde. Cadernos de Saúde Pública, Rio de Janeiro, v. 20, n. 2, p. 190-198, 2004.

Recebido em: 08 fev. 2012

Aprovado em: 16 jun. 2012

Endereço para correspondência

Mathias Roberto Loch

Departamento de Educação Física -

Centro de Educação Física e Esporte

Universidade Estadual de Londrina

Rod. Celso Garcia Cid PR 445 -

Campus Universitário Londrina-PR, Brasil.

CEP 8605।-980 